\title{
Mediastinal staging for lung cancer
}

\author{
Jacob Gelberg MD FRCPC ${ }^{1}$, Sean Grondin MD MPH FACS FRCSC ${ }^{2}$, Alain Tremblay MDCM FRCPC FCCP ${ }^{3}$
}

\author{
J Gelberg, S Grondin, A Tremblay. Mediastinal staging for lung \\ cancer. Can Respir J 2014;21(3):159-161.
}

Staging of the mediastinal and hilar lymph nodes plays a crucial role in identifying the best treatment option for patients with confirmed or suspected lung cancer and, in many cases, can simultaneously confirm a diagnosis of cancer. Noninvasive modalities, such as computed tomography (CT), positron emission tomography (PET) and PET-CT, are an important first step in this assessment. Ultimately, invasive staging is frequently required to confirm or rule out the presence of metastatic disease within the lymph nodes. The present focused review describes and compares noninvasive and invasive modalities for mediastinal staging in lung cancer.

Key Words: Bronchoscopy; Endobronchial ultrasound; Lung cancer; Mediastinoscopy; Positron emission tomography

taging of lung cancer, while primarily a prognosis-based system, is Sessential to select the best treatment strategy for a given patient. Mediastinal and hilar lymph node status are a key component of the TNM staging system and, in many cases, determines whether surgical resection will be performed. Invasive staging modalities can often simultaneously confirm diagnosis and offer specimens for molecular testing and should, therefore, be considered early in the evaluation of a patient with suspected lung cancer. We review noninvasive and invasive mediastinal staging modalities for lung cancer and offer recommendations for their application in clinical practice. In selecting data from the published literature, we emphasized international guideline documents, high-quality randomized trials and meta-analyses.

\section{NONINVASIVE STAGING}

Following a thorough history, physical examination and chest radiography, computed tomography (CT) of the chest and upper abdomen with intravenous contrast is usually performed. CT provides detailed anatomical information about the primary lesion, lymph node size and location, as well as assessing common metastatic sites. Any lymph node with a short-axis diameter $\geq 1 \mathrm{~cm}$ is considered to be abnormal (1). The median sensitivity and specificity of CT for identifying mediastinal lymph node metastasis are $55 \%$ and $81 \%$, respectively, when the prevalence of disease is $30 \%$ (1) (Table 1). Unfortunately, this diagnostic accuracy is insufficient to guide treatment decisions.

Positron emission tomography (PET) relies on the premise that lung cancer cells demonstrate an increased rate of glucose absorption and glycolysis compared with normal tissue leading to increased radiotracer (fluorodeoxyglucose ${ }^{18} \mathrm{~F}$ ) accumulation in malignant cells (1). The median sensitivity and specificity of PET for detecting lymph node metastases is $80 \%$ and $88 \%$, respectively, when the prevalence of metastasis is $28 \%$ (1) (Table 1). PET has been shown to be more accurate than CT at predicting mediastinal metastasis (1). PET can also provide additional information regarding the primary lung lesion and detect metastatic disease outside the thorax. The poor spatial resolution of PET scanning can be improved by dual-modality PET-CT. Studies examining this imaging technique report a slightly higher specificity of $90 \%$ but a lower sensitivity of $62 \%$ based on a systematic review of 19 studies including 2014 patients (1). Randomized trials have demonstrated a $50 \%$ reduction in the number of unnecessary

\section{La classification des ganglions médiastinaux en cas de cancer du poumon}

La classification des ganglions médiastinaux et des ganglions hilaires joue un rôle essentiel pour déterminer la meilleure option thérapeutique chez les patients atteints d'un cancer du poumon confirmé ou présumé et, dans bien des cas, peut également confirmer un diagnostic de cancer. Des modalités non effractives, telles que la tomodensitométrie, la tomographie par émission de positrons et la tomodensitométrie par émission de positrons, sont des premières étapes importantes de cette évaluation. Au bout du compte, il faut souvent recourir à une classification effractive pour confirmer ou écarter la présence d'une maladie métastatique des ganglions lymphatiques. La présente analyse ciblée décrit et compare les modalités non effractives aux modalités effractives pour classer les ganglions médiastinaux en cas de cancer du poumon.

\section{TABLE 1}

Lymph node staging modalities

\begin{tabular}{|c|c|c|c|c|}
\hline \multirow[b]{2}{*}{ Staging modality } & \multirow{2}{*}{$\begin{array}{c}\text { Lymph node } \\
\text { access }\end{array}$} & \multicolumn{3}{|c|}{$\%$} \\
\hline & & Sensitivity* & Specificity* & $\mathrm{NPV}^{*}$ \\
\hline \multicolumn{5}{|l|}{ Noninvasive } \\
\hline CT chest & All & 55 & 81 & 83 \\
\hline PET & All & 80 & 88 & 91 \\
\hline PET-CT & All & 62 & 90 & 90 \\
\hline \multicolumn{5}{|l|}{ Invasive } \\
\hline Mediastinoscopy ${ }^{\dagger}$ & $2 \mathrm{R}, 2 \mathrm{~L}, 4 \mathrm{R}, 4 \mathrm{~L}, 7$ & 78 & $100^{\ddagger}$ & 91 \\
\hline EBUS ${ }^{\dagger}$ & $\begin{array}{c}2 \mathrm{R}, 2 \mathrm{~L} \text { 4R, 4L, 7, 10R, } \\
\text { 10L, 11R, 11L }\end{array}$ & 89 & $100^{\ddagger}$ & 91 \\
\hline EUS & $4 \mathrm{~L}, 7,5,8,9$ & 89 & $100^{\ddagger}$ & 86 \\
\hline $\begin{array}{l}\text { Combined } \\
\text { EBUS/EUS }\end{array}$ & $\begin{array}{c}2 \mathrm{R}, 2 \mathrm{~L} 4 \mathrm{R}, 4 \mathrm{~L}, 7,10 \mathrm{R} \\
10 \mathrm{~L}, 11 \mathrm{R}, 11 \mathrm{~L}, 5,8,9\end{array}$ & 91 & $100^{\ddagger}$ & 96 \\
\hline
\end{tabular}

*Sensitivity, specificity and negative predictive value (NPV) for each staging modality were obtained from studies that may have different patient populations and direct comparison between modalities based on these values alone should be avoided; 'Studies directly comparing endobrachial ultrasound

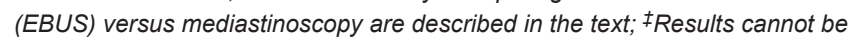
verified in studies reporting $100 \%$ specificity because no follow-up test was performed to confirm the results; Source: American College of Chest Physicians lung cancer guidelines, 3rd edn (1). CT Computed tomography; EUS Endoscopic ultrasound; PET Positron emission tomography

thoracotomies resulting from the use of preoperative PET or PET-CT scanning $(2,3)$. As such, PET or PET-CT imaging has become standard in most patients being considered for surgical or more aggressive chemoradiation protocols. The limited utility of PET modalities in patients with early asymptomatic disease (peripheral stage cla lesion $<3 \mathrm{~cm}$, no enlarged mediastinal nodes on CT) or clearly advanced disease does not justify its routine use.

Clinicians should remember that incorrect upstaging and falsepositive results can occur with PET-CT. Cytopathological sampling of positive PET-CT findings is recommended before finalizing treatment decisions.

\footnotetext{
${ }^{1}$ Department of Medicine, McMaster University, Hamilton, Ontario; ${ }^{2}$ Department of Surgery; ${ }^{3}$ Department of Medicine, University of Calgary, Calgary, Alberta

Correspondence: Dr Alain Tremblay, Department of Medicine, University of Calgary, 3330 Hospital Drive Northwest, Calgary, Alberta T2N 4N1.

Telephone 403-210-3866, fax 403-944-1577, e-mail alain.tremblay@ucalgary.ca
} 

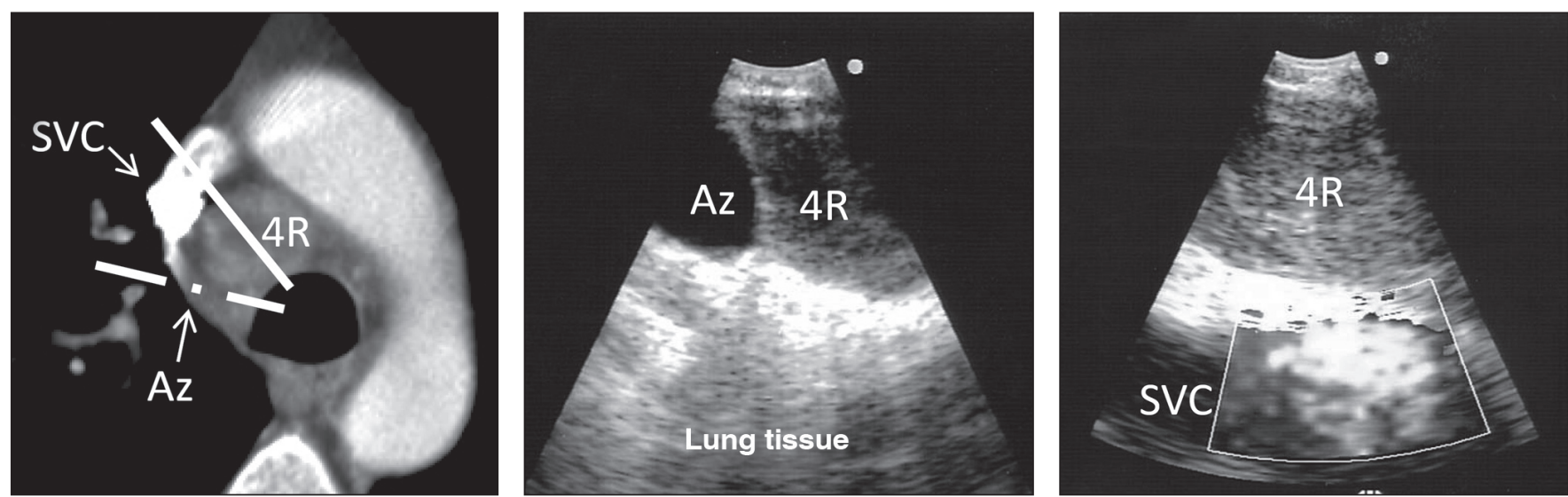

Figure 1) Right lower paratracheal (4R lymph node), superior vena cava (SVC) and azygous vein (Az) on computed tomography (left) and endobrachial ultrasound (centre, right)

\section{SURGICAL STAGING}

Cervical mediastinoscopy has been the gold standard for preoperative invasive mediastinal lymph node staging for lung cancer patients. It is performed under general anesthesia and most patients are discharged home the same day. Mediastinoscopy provides access to upper (\#2) and lower $(\# 4)$ paratracheal, as well as subcarinal $(\# 7)$ nodes (Table 1$)$. Relative contraindications include previous mediastinoscopy, radiation therapy and cervical arthritis (4). In a systematic review (1), mediastinoscopy had a median sensitivity of $78 \%$ and a negative predictive value (NPV) of $91 \%$. Complications are rare and include pneumothorax, recurrent laryngeal nerve damage, vascular injury and tracheal laceration, with an overall morbidity of $1.07 \%$ and mortality of $0.05 \%$ (5). The false-negative rate for mediastinoscopy is reported to be $5.5 \%$, with most false negatives occurring in lymph nodes that could not be accessed for biopsy such as the posterior subcarinal nodes (5). Anterior mediastinoscopy (Chamberlain procedure) can be used to access aortopulmonary (\#5) and para-aortic (\#6) nodes. Videoassisted thoracic surgery can also be used to biopsy $\# 5$ and $\# 6$ nodes in addition to paraesophageal (\#8), pulmonary ligament nodes (\#9) and ipsilateral (\#11) hilar lymph nodes.

The use of extended cervical mediastinoscopy or 'super mediastinoscopy', such as transcervical extended mediastinal lymphadenectomy and video-assisted mediastinoscopic lymphadenectomy, has been described. These procedures aim for the complete removal of bilateral mediastinal lymph node stations with surrounding adipose tissue to improve the accuracy of staging. Currently, the use of these procedures is controversial and confirmatory studies examining the safety and accuracy of these techniques are required (6).

\section{ENDOSCOPIC ULTRASOUND}

Endobronchial ultrasound (EBUS)-guided transbronchial needle aspiration and endoscopic ultrasound (EUS)-guided fine needle aspiration have emerged as viable alternatives to mediastinoscopy owing to their excellent sensitivity and ability to access multiple lymph node stations in a minimally invasive fashion (1). These are performed using specialized endoscopes with a curvilinear ultrasound probe at its tip. Lymph nodes are identified and sampled using a needle device under direct visualization in real time. Either test can be performed in an outpatient setting under conscious sedation with low complication rates.

EBUS offers excellent access to mediastinal, hilar and interlobar lymph nodes most commonly involved in lung cancer (Table 1, Figure 1). Lacking is access to prevascular (\#3a), aortopulmonary $(\# 5)$, para-aortic (\#6), paraesophageal (\#8) and pulmonary ligament (\#9) nodes. A large number of publications recently summarized in a meta-analysis (7) have demonstrated excellent sensitivity and NPV of EBUS. A recent review of 2756 patients demonstrated overall median sensitivity of $89 \%$ and NPV of $91 \%$ (Table 1) (1).
Complications of EBUS are similar to those of bronchoscopy and conventional transbronchial needle aspirate and include rare instances of pneumothorax and mediastinitis (7). EUS offers some technical advantages compared with EBUS such as higher quality and wider field of ultrasound image and less air artefact (4). The needle tends to pass more easily through soft esophageal tissue compared with the cartilaginous airway wall. EUS can sample lymph nodes not normally accessible by EBUS or mediastinoscopy, including stations 8, 9 and 5, as well as providing access to stations $4 \mathrm{~L}$ and 7 (Table 1) (4). Inability to access hilar right paratracheal (\#4R) nodes as well as the primary lesion are drawbacks. Recent studies of patients undergoing EUS demonstrate a sensitivity and accuracy of $83 \%$ and $89 \%$, respectively $(1,8,9)$.

Combination of EBUS and EUS allows near-complete access to all the lymph nodes of the mediastinum; the use of these complementary techniques has been evaluated in several studies $(10,11)$. A review of the published literature demonstrates a median sensitivity of $91 \%$ and NPV of $86 \%$ (1). A recent meta-analysis identified only two serious complications (pneumothorax and lymph node abscess) of 822 lung cancer patients (12). Published studies consistently demonstrate a higher sensitivity and NPV when combined EBUS and EUS are compared with either modality alone (11), although this may simply be a result of more needle passes performed rather than a true complementary value of each technique. This combined endoscopic approach comes at a cost of increased procedure time, need for additional training and equipment (11). It is unclear whether these costs are justified because these studies did not demonstrate a shift in stage of disease or lead to change in management when combined EBUS and EUS were compared with each modality individually.

\section{COMPARATIVE TRIALS}

Several trials have compared endoscopic approaches with cervical mediastinoscopy. A Canadian study prospectively enrolled 159 patients with confirmed or suspected lung cancer and performed EBUS immediately followed by cervical mediastinoscopy under general anesthesia in all patients, finding no significant differences in sensitivity (81\% versus $79 \%$ ), NPV (91\% versus 90\%) or accuracy (93\% versus 93\%) between the two tests, but higher complications in the mediastinoscopy group (12). A multicentre study randomly assigned 241 patients to EBUS and EUS followed by mediastinoscopy if no nodal metastasis was identified versus mediastinoscopy alone and found no difference in sensitivity and NPV between mediastinoscopy and combined EBUS and EUS (13). It should be noted that the majority of the published experience with EUS staging of lung cancer has included subjects with moderate or higher prevalence of mediastinal disease (>35\%). Uncertainty remains with regard to application of this and other staging modalities in cohorts with lower prevalence of disease (eg, CT- or PET/CT-negative mediastinum). 
Comparisons between EBUS and EUS showed identical sensitivity in 150 patients with known or suspected lung cancer (11). A recent randomized trial found significant improvement in sensitivity, accuracy and upstaging when EUS was followed by EBUS, while the converse was not observed (14). This study suggests that EUS provides minimal additional diagnostic gain when EBUS is performed first, and that perhaps EBUS alone is sufficient for adequate endoscopic staging of the mediastinum when node stations $\# 5, \# 8$ and $\# 9$ are not suspected to be involved with disease on imaging (14).

\section{PRACTICAL APPROACH AND RECOMMENDATIONS}

Staging with PET or PET-CT should be performed in patients with proven or suspected non-small cell lung cancer who are being considered for surgical or aggressive chemoradiation protocols. Cytopathological confirmation of abnormal PET findings that may impact treatment is critical to avoid inappropriate exclusion of curative treatment due to false-positive PET results. Patients with apparent peripheral early stage (Ia) disease on CT or those with clear evidence of advanced disease or metastasis on CT do not require a PET scan.

Given their high sensitivity and low invasiveness, EUS staging modalities should be considered before surgical techniques when mediastinal involvement is suspected (ie, enlarged lymph nodes on CT chest and/or PET-positive lymph nodes) (1). Nevertheless, if negative in this setting, more definitive surgical staging should be considered before tumour resection. Application of EUS techniques can also be considered in cases with lower probability of mediastinal disease (CT and/or PET negative but with central mass or positive N1 nodes). Although it remains the best first test in these patients (1), it should only be applied if negative results are considered acceptable to determine resectability by the local treatment team. If surgical mediastinoscopy is still considered necessary before resection in a given patient, proceeding to this test first may be more efficient. Cases with very low probability of mediastinal disease (peripheral stage Ia on CT and PET) do not require preoperative invasive mediastinal staging.

Both diagnosis and staging for suspected non-small cell lung cancer should proceed simultaneously and in a coordinated fashion. According

\section{REFERENCES}

1. Silvestri GA, Gonzales AV, Jantz MA, et al. Methods of staging non-small cell lung cancer: Diagnosis and management of lung cancer, 3rd edn. American College of Chest Physicians Evidence-Based Clinical Practice Guidelines. Chest 2013;143(Suppl):e211S-e250S.

2. van Tinteren H, Hoekstra OS, Smit EF, et al. Effectiveness of positron emission tomography in preoperative assessment of patients with suspected non-small cell lung cancer: The PLUS multicentre randomized trial. Lancet 2002;359:1388-93.

3. Fischer B, Lassen, U, Mortensen, J et al. Preoperative staging of lung cancer with combined PET-CT. N Engl J Med 2009;361:32-9.

4. Block MI, Tarrazzi FA. Invasive mediastinal staging: Endobronchial ultrasound, endoscopic ultrasound, and mediastinoscopy. Semin Thorac Surg 2013;25:218-27.

5. Lemaire A, Nikolic I, Petersen T, et al. Nine-year single center experience with cervical mediastinoscopy: Complications and false negative rate. Ann Thorac Surg 2006;82:1185-90.

6. Yendamuri S, Demmy TL. IsVAMLA/TEMLA the new standard of preresection staging of non-small cell lung cancer? J Thorac Cardiovasc Surg 2012;144;S14-17.

7. Dong X, Qiu X, Liu Q, et al. Endobronchial ultrasound-guided transbronchial needle aspiration in the mediastinal staging of non-small cell lung cancer: A meta-analysis. Ann Thorac Surg 2013;96:1502-7.

8. Annema JT, Bohoslavsky R, Burgers S, et al. Implementation of endoscopic ultrasound for lung cancer staging. Gastrointest Endosc 2010;71:64-70. to the guidelines, minimally invasive endoscopic tests for mediastinal staging are the first best test and will often confirm stage and diagnosis directly (1). A recent study demonstrated guideline-consistent care in only $22 \%$ of patients undergoing staging for lung cancer resulting in more invasive tests per patient and a higher complication rate $(0 \%$ versus $17 \% ; \mathrm{P}=0.01$ ) largely due to the use of $\mathrm{CT}$-guided biopsy as the first invasive diagnostic test (15). Adherence to guidelines in patients with suspected lung cancer and mediastinal lymphadenopathy will reduce both complications and health care costs.

\section{CONCLUSIONS}

Staging of mediastinal and hilar lymph nodes plays a crucial role in identifying the best treatment option for patients with confirmed or suspected lung cancer without distant metastasis. Imaging techniques, such as CT, PET and PET-CT, are an important step in evaluating lymph node involvement but ultimately do not have the required diagnostic accuracy for making the final treatment decision. Invasive lymph node staging techniques, such as EBUS and/or EUS, are recommended in most patients without extrathoracic metastatic disease and, in many cases, will also confirm a diagnosis of cancer. The gold standard of mediastinoscopy for invasive staging is challenged by these EUS techniques, which are now considered to be the initial invasive test in most instances for which lymph node staging is required. Adherence to this guideline-recommended approach reduces complications and health care costs. Combination of EBUS and EUS may offer a higher sensitivity and NPV compared with either modality alone. Further studies are needed to determine whether combining these two modalities may improve the accuracy of clinical staging leading to more accurate treatment decisions.

DISCLOSURES: Drs J Gelberg and S Grondin have no financial disclosures or conflicts of interest to declare. Dr A Tremblay has received consulting fees from Spiration / Olympus America, which manufactures and sells endoscopic ultrasound systems.

9. Micames CG, McCrory DC, Pavey DA, et al. Endoscopic ultrasound-guided fine-needle aspiration for non-small cell lung cancer staging: A systematic review and metaanalysis. Chest 2007;131:539-48

10. Hwangbo B, Lee GK, Lee HS, et al. Transbronchial and transesophageal fine-needle aspiration using an ultrasound bronchoscope in mediastinal staging of potentially operable lung cancer. Chest 2010;138:795-802.

11. Wallace MB, Pascual JMS, Raimondo M, et al. Minimally invasive endoscopic staging of suspected lung cancer. JAMA 2008;299:540-6.

12. Yasufuku K, Pierre A, Darling G, et al. A prospective controlled trial of endobronchial ultrasound-guided transbronchial needle aspiration compared with mediastinoscopy for mediastinal lymph node staging for lung cancer. J Thorac Cardiovasc Surg 2011;142:1393-400

13. Annema JT, van Meerbeeck JP, Rintoul RC, et al. Mediastinoscopy vs endosonography for mediastinal lymph node staging of lung cancer: A randomized trial. JAMA 2010;304:2245-52.

14. Kang HJ, Hwangbo B, Lee G-K, et al. EBUS-centred versus EUScentred mediastinal staging in lung cancer: Randomized controlled trial. Thorax 2014;69:261-8.

15. Almeida FA, Casal RF, Jimenez CA, et al. Quality gaps and comparitive effectiveness in lung cancer staging: The impact of test sequencing on outcomes. Chest 2013;144:1776-82. 


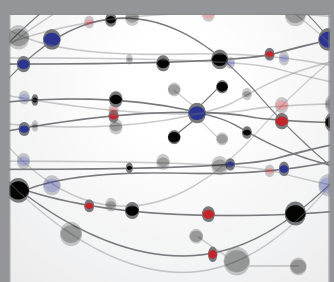

The Scientific World Journal
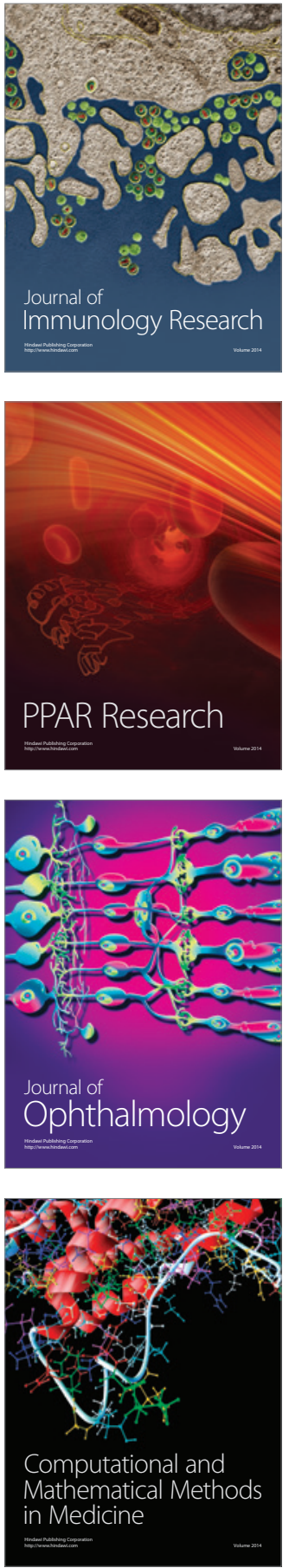

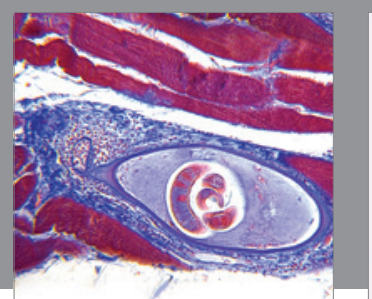

Gastroenterology Research and Practice

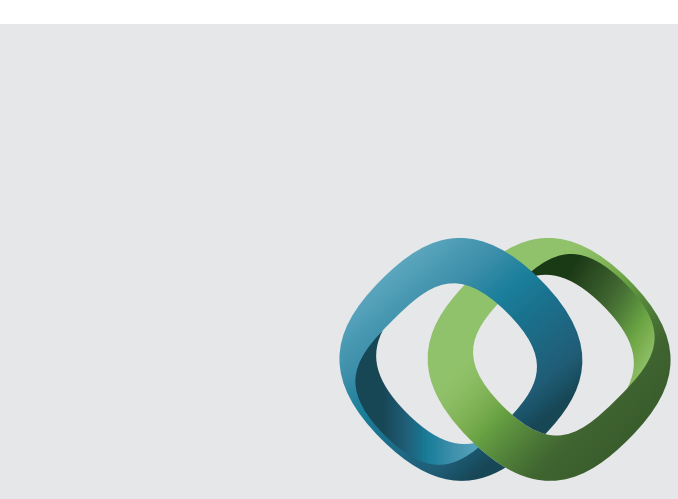

\section{Hindawi}

Submit your manuscripts at

http://www.hindawi.com
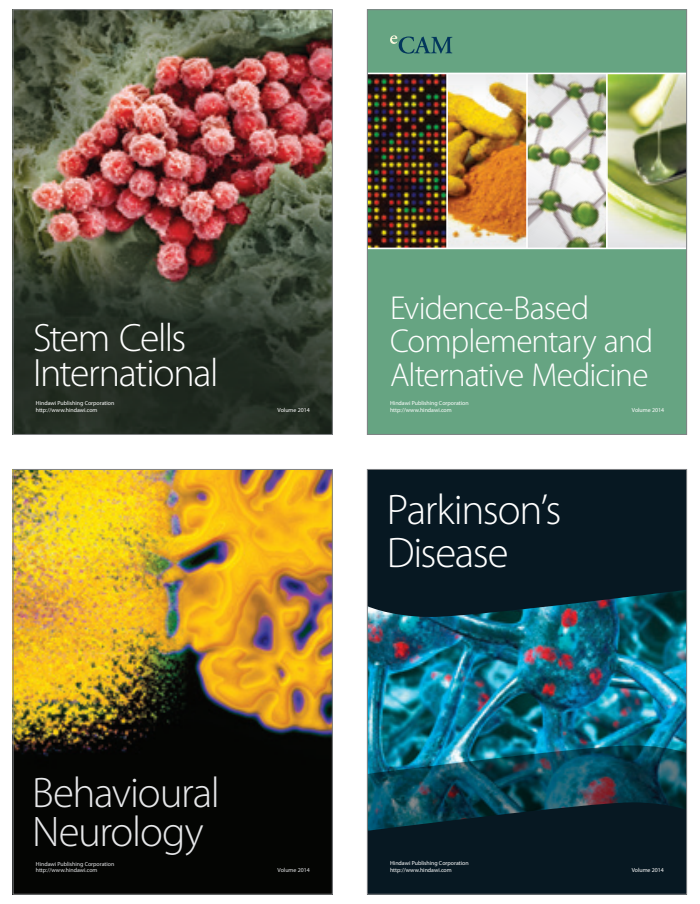
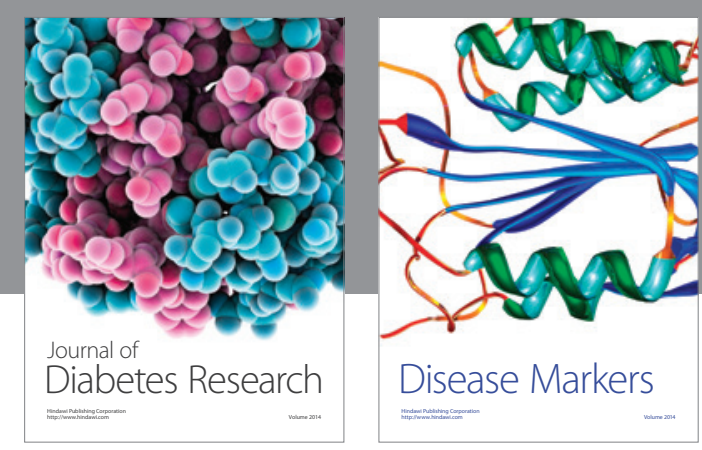

Disease Markers
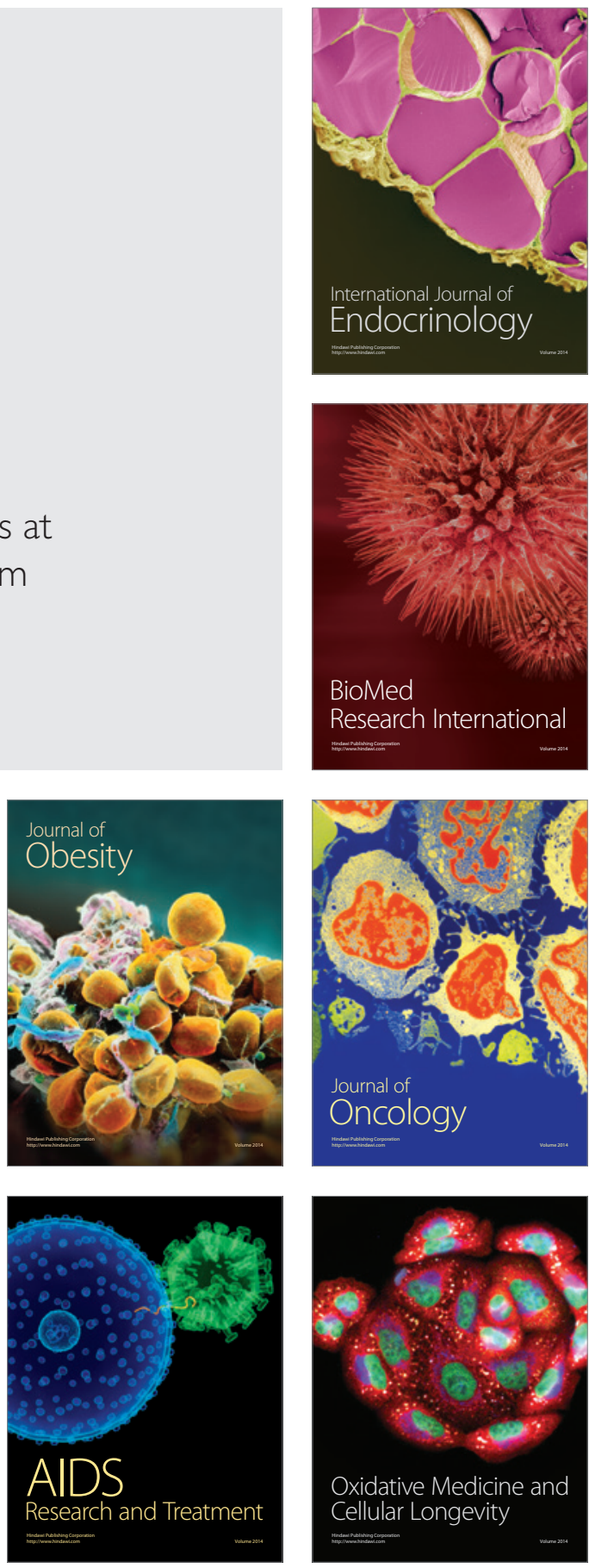